\title{
MST1: a promising therapeutic target to restore functional beta cell mass in diabetes
}

\author{
Amin Ardestani ${ }^{1} \cdot$ Kathrin Maedler $^{1}$
}

Received: 16 November 2015 / Accepted: 23 December 2015 / Published online: 6 April 2016

(C) Springer-Verlag Berlin Heidelberg 2016

\begin{abstract}
The loss of insulin-producing beta cells by apoptosis is a hallmark of all forms of diabetes mellitus. Strategies to prevent beta cell apoptosis and dysfunction are urgently needed to restore the insulin-producing cells and to prevent severe diabetes progression. We recently identified the serine/ threonine kinase known as mammalian sterile 20-like kinase 1 (MST1) as a critical regulator of apoptotic beta cell death and dysfunction. MST1 activates several apoptotic signalling pathways, which further stimulate its own cleavage, leading to a vicious cycle of cell death. This led us to hypothesise that MST1 signalling is central to the initiation of beta cell death in diabetes. We found that MST1 is strongly activated in a diabetic beta cell and induces not only its death but also directly impairs insulin secretion through promoting proteasomal degradation of key beta cell transcription factor, pancreatic and duodenal homeobox 1 (PDX1), which is critical for insulin production.

Pre-clinical studies in various animal models of diabetes have reported that MST1 deficiency remarkably restores normoglycaemia and beta cell function and prevents the development of diabetes. Importantly, MST1 deficiency can revert fully diabetic beta cells to a non-diabetic state. MST1 may serve as a target for the development of novel therapies for diabetes that trigger the cause of the disease, namely, the destruction of the beta cells. The major current focus of our investigation is to
\end{abstract}

Amin Ardestani

ardestani.amin@gmail.com

Kathrin Maedler

kmaedler@uni-bremen.de

1 Islet Biology Laboratory, Centre for Biomolecular Interactions Bremen, University of Bremen, Leobener Straße NW2, Room B2080, 28359 Bremen, Germany identify and test the efficacy of potent inhibitors of this death signalling pathway to protect beta cells against the effects of autoimmune attack in type 1 diabetes and to preserve beta cell mass and function in type 2 diabetes. This review summarises a presentation given at the 'Can we make a better beta cell?' symposium at the 2015 annual meeting of the EASD. It is accompanied by two other reviews on topics from this symposium (by Heiko Lickert and colleagues, DOI: 10.1007/s00125016-3949-9, and by Harry Heimberg and colleagues, DOI: 10. 1007/s00125-016-3879-6) and a commentary by the Session Chair, Shanta Persaud (DOI: 10.1007/s00125-016-3870-2).

Keywords Apoptosis $\cdot$ Beta cells $\cdot$ Diabetes $\cdot$ Hippo pathway · Insulin · Islets · Mammalian sterile 20-like kinase · MST1 · PDX1 - Review

$\begin{array}{ll}\text { Abbreviations } \\ \text { BIM } & \text { Bcl-2-like protein 11 } \\ \text { JNK } & \text { c-Jun N-terminal kinase } \\ \text { LATS } & \text { Large tumor suppressor } \\ \text { MST } & \text { Mammalian sterile 20-like kinase } \\ \text { PDX1 } & \text { Pancreatic and duodenal homeobox 1 } \\ \text { YAP } & \text { Yes-associated protein }\end{array}$

There is ongoing debate regarding whether beta cell death or impaired beta cell function plays the bigger role in the pathology of diabetes. While it is clear that the specific destruction of the beta cell by the activated immune cells is the cause of type 1 diabetes, loss of function due to beta cell overwork during compensation states, e.g. in obesity, seems to occur earlier than the full collapse of the infrastructure of the cell and apoptotic cell death in the context of type 2 diabetes. But even prior to the development of type 2 diabetes, i.e. in patients 
with impaired glucose tolerance, beta cell loss is apparent and correlates with elevated fasting glucose levels [1]. Beta cell death is consistently evident in at-risk individuals prior to the diagnosis of type 1 diabetes, indicating the importance of beta cell loss in the process of disease progression [2].

For a therapy to be successful, both loss of function and beta cell death need to be targeted. If loss of function occurs earlier, possibly before any beta cell death, diabetes progresses and it is that ultimately leads to beta cell loss. Thus, targeting the one (function) or the other (survival), may not help to restore functional beta cells. It became clear that beta cell death is causative for both types of diabetes [3, 4]. Thus, the elucidation of the molecular processes associated with beta cell apoptosis and maintenance of beta cell mass is critical for the development of new therapeutic strategies aimed at delaying and/or preventing these pathological events. At present there are no treatment strategies that preserve the endogenous beta cell mass. Inhibiting pro-apoptotic, or potentiating anti-apoptotic, signalling could represent a potential strategy for ensuring beta cell survival in diabetes. Despite the large amount of evidence supporting the essential role of beta cell apoptosis in the loss of functional beta cells and diabetes, to date, the specific proapoptotic signalling pathways have not been comprehensively studied. Using a hypothesis-based approach, we aimed to identify a pro-diabetic-driven apoptotic pathway that is specifically switched on during beta cell failure in isolated human islets exposed to a complex diabetic milieu, in islets of pancreatic sections obtained at autopsy from patients with type 2 diabetes and in islets of diabetic mouse models. In a recent study we demonstrated that the kinase mammalian sterile 20-like kinase 1 (MST1) is activated by multiple diabetogenic conditions in vitro and in vivo [5]. MST1 overexpression itself leads to impaired beta cell survival and function. In the remainder of this review we will present and discuss the effects of MST1 and the pathways it regulates in the beta cell; MST1 impairs beta cell function and exerts apoptosis-inducing effects through at least two mechanisms: (1) by specifically activating the intrinsic pathway of cell death through the upregulation of the mitochondrial pro-apoptotic protein Bcl-2-like protein 11 (BIM), which leads to beta cell apoptosis, and (2) by directly phosphorylating the critical beta cell transcription factor pancreatic and duodenal homeobox 1 (PDX1) on Thr-11, resulting in the ubiquitination and degradation of PDX1, which leads to the impairment beta cell function.

\section{MST1: core component of the Hippo signalling pathway}

The Hippo pathway has emerged as a complex signalling network that has significant implications for our understanding of the molecular mechanisms of cancer, development and stem cell biology [6]. First discovered by genetic screens in
Drosophila, the conservation of this pathway in mammals has been firmly established [6]. The kinase cascade of MST1/2 and large tumour suppressors 1 and 2 (LATS1/2) represent core components of the mammalian Hippo pathway. MST1/2, in complex with the regulatory protein Salvador (Sav1), phosphorylate and activate LATS1/2, which also form a complex with the regulatory protein Mps-one binder 1 (MOB1). The transcriptional co-activator Yes-associated protein (YAP) is a major downstream effector of the Hippo pathway. LATS1/2 inhibits YAP by direct phosphorylation at Ser-127, which results in the binding of YAP to 14-3-3 proteins, leading to its cytoplasmic sequestration and its ubiquitin-dependent degradation, which thereby prevents YAP transcriptional activity $[7,8]$. YAP acts mainly through the TEA domain (TEAD) family of transcription factors to promote the expression of target genes that modulate diverse cellular functions, such as proliferation and apoptosis. The loss of any component of the kinase core results in a YAP-dependent increase in proliferation and resistance to apoptosis in multiple tissues, establishing Hippo as a powerful tool for regulating organ size [7, 8]. As an emerging significant player in many diseases, the Hippo pathway has attracted increasing attention as a target for new drugs. While the role of Hippo signalling in tumour development gains increasing attention $[8,9]$, non-cancer related abnormalities involving Hippo components have only been studied to a limited extent $[5,10]$. The regulation of the Hippo pathway by multiple signals is not surprising, given the remarkable role of this pathway in cell proliferation and apoptosis [11]. Interestingly, several diabetes-related pathways have been reported to crosstalk with Hippo signalling $[5,12,13]$.

Full-length MST1 is not only expressed in beta cells, it is ubiquitously expressed and involved in multiple cellular processes, such as morphogenesis, proliferation, the stress response and apoptosis $[14,15]$. When activated in the cellular response to cytotoxic stress, MST1 mediates cell death triggered by a variety of apoptotic stimuli, such as oxidative stress, FasL treatment, TNF- $\alpha$, growth factor withdrawal and genotoxic drugs $[14,15]$. The effects of MST1 can be nicely explained by its structure: MST1 contains an Nterminal kinase catalytic domain, followed by a non-catalytic tail that contains two caspase cleavage sites [16, 17], two nuclear export sites (NES) and dimerisation domains at the C-terminal. Activated MST1 forms dimers via the Salvador/ Rassf/Hippo (SARAH, aa 433-480) domain at the extreme Cterminal region of MST1, which is autophosphorylated on specific threonine residues in the activation loop $[16,17]$.

\section{MST1 potentiates beta cell apoptosis through a caspase-mediated activation loop}

MST1 plays a central role in the initiation of cell death [14-17, 20]. Caspase-mediated cleavage of MST1 results in the 
removal of the C-terminal inhibitory domain and subsequent translocation of the constitutively active catalytic fragment to the nucleus $[16,17]$. This cleaved fragment comprising the Nterminal kinase domain is nearly ten times more active than the full-length enzyme $[14,16,17]$. Translocation of the kinase domain from the cytosol to the nucleus presumably offers a different selection of substrates to phosphorylate [18]. The mutation of these caspase cleavage sites attenuates the kinase activity of MST1, its nuclear translocation and its ability to induce apoptosis $[16,17]$. Under resting conditions, MST1 is exclusively located in the cytoplasm.

We have reported that pro-diabetic stimuli lead to MST1 auto-phosphorylation and activation of caspases in pancreatic beta cells (initiation phase of apoptosis) [5]. Autophosphorylated MST1 is active and phosphorylates multiple substrates that amplify the apoptotic signal, further increasing caspase activation. In this scenario, MST1-triggered mitochondrial pro-apoptotic BIM. BIM activation integrates signals from pro-apoptotic diabetogenic stimuli to the caspase machinery. In addition, active caspases (namely, caspase-3) cleave off the C-terminal inhibitory domain of MST1 and liberate the $\mathrm{N}$-terminal fragment. The fact that the fragment has much higher activity than the full-length MST1 explains how the cleavage of such a tiny proportion of total cell MST1 has a big effect on its activation. This positive feedback in the pathway to amplifies caspase-3 activation and boosts transmission of the apoptotic signal (amplification phase of apoptosis). It is possible that such an internal activation loop between MST1 and the caspase machinery mediates apoptosis in the beta cell under conditions of chronic stress (see Fig. 1). For example, in response to short exposure to glucose, beta cells neither undergo apoptosis nor show any signs of MST1 activation. This situation only switches under conditions of chronic glucotoxicity, where MST1 activation occurs together with caspase cleavage as a measure for apoptosis [5].

MST1 alone sufficiently induces caspase-3 activation (apoptosis initialisation) as well as apoptosis amplification through a caspase-mediated positive feedback loop under diabetogenic conditions, These findings suggest the involvement of MST1 in pre- and post-caspase activation events in beta cells.

\section{MST1-regulated signalling pathways in beta cells}

Protein kinase activities are altered during apoptosis in a variety of cell types, indicating that protein phosphorylation is involved in apoptotic regulation [19]. Among these, MST1 serves as the key component in regulating apoptotic entry in several cell types under specific conditions through the phosphorylation-dependent regulation of multiple substrates [15]. The broad nature of the diabetic stimuli and multiple cell systems used in our study in which we found activation of
MST1 suggests that this enzyme may be a common component of the diverse signalling pathways leading to beta cell apoptosis. From a therapeutic perspective, it is important to determine whether increased MST1 activity is a general mechanism in various diabetic conditions, since next-generation drugs for type 1 and type 2 diabetes need to aim to preserve functional beta cell mass, and thus must target more than a single apoptotic pathway. Using a complex diabetic milieu that includes proinflammatory cytokines, gluco- and lipotoxicity and oxidative stress in vitro, we demonstrated that MST1 is activated in beta cells in response to a broad range of stimuli. While we have already identified direct upstream regulators of MST1 which modulate the effect of diabetogenic conditions on MST1 activation and subsequent apoptosis (A. Ardestani, K. Maedler; unpublished results), the multiple downstream targets of MST1 are well established, e.g. the LATS1/2, histone H2B, FOXO members family, as well as induction of stress kinase c-Jun-N-terminal kinase (JNK) and caspase-3 activation [15]. Most of them occur in the stressed beta cell, and we have recently shown that LATS2 is a potent inducer of beta cell apoptosis [21]. This is consistent with the multi-factorial nature of beta cell failure in diabetes on one hand and the multiple regulatory features and cell type-specific mechanisms of MST1 on the other hand, which may be required to integrate inputs from diverse signalling pathways to an apoptotic end.

JNK phosphorylation is a strong signal for beta cell destruction and its inhibition has been discussed as a potent strategy to rescue the beta cell, as well as to promote insulin sensitivity under proinflammatory conditions $[22,23]$. The phosphatidylinositol 3-kinase (PI3K)-AKT pathway is a potent suppressor of JNK in islets, and serves as an antiapoptotic tool [24]. AKT is a main downstream effector of PI3K, whose pleiotropic functions lead to the inhibition of apoptosis $[25,26]$. The actions of AKT are also mediated through the inhibition of MST1, preventing its apoptosispromoting effects $[27,28]$. AKT phosphorylates MST1 at Thr-120 and Thr-387, leading to inhibition of its kinase activity, nuclear translocation, autophosphorylation and ability to induce apoptosis. The observation that MST1 and AKT1 are localised to identical subcellular compartments in human prostate tumours suggests that MST1 may be an important branch pathway of PI3K-AKT. MST1 was identified as a component of an AKT1 multi-protein complex isolated from lipid raft-enriched fractions of human prostate cancer cells [20]. Both MST1 and its active cleaved forms physically interact with AKT1 and function as direct inhibitors of AKT1. Depletion of MST1 or MST2 with siRNA increases AKT activity, and depletion of both proteins further enhances AKT activity [20]. We have confirmed that MST1-induced apoptosis is regulated by AKT1 in islets [5]. Activation of the PI3K-AKT pathway through the addition of exogenous mitogens or a constitutively active form of AKT1 abrogates 


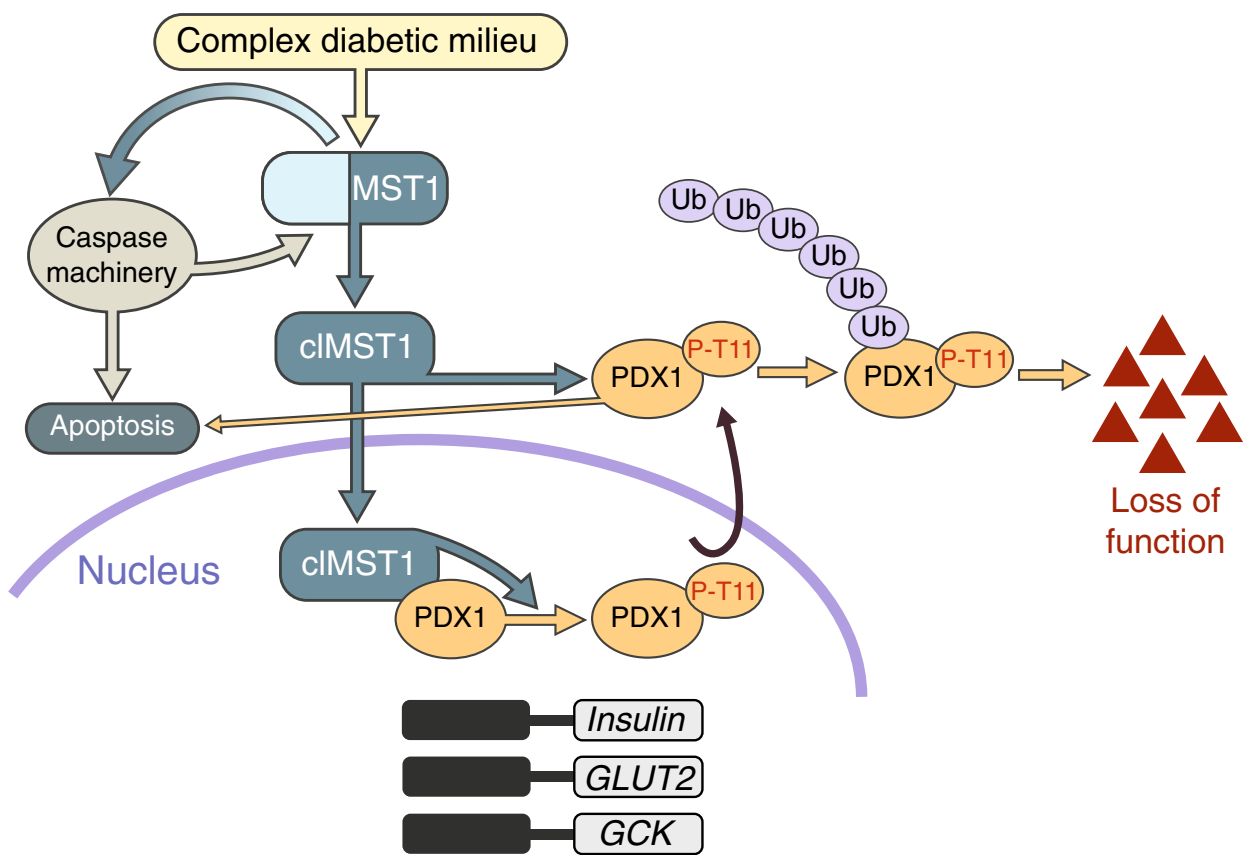

Fig. 1 Diabetic stimuli lead to activation of MST1, apoptosis and loss of function. Diabetogenic conditions switch on the MST1 pro-apoptotic machinery in beta cells, either directly or through activation pathways that are currently under investigation. Active MST1 triggers the caspase machinery, which induces caspase-dependent cleavage of MST1 (clMST1) to its constitutively active fragment, which leads to a potentiation of MST1 activation and increased kinase activity by a positive feedback mechanism, ending in an acceleration of beta cell death.
Cleaved MST1 translocates to the nucleus and directly phosphorylates PDX1 on Thr-11 (P-T11; we do not exclude the possibility that MST1 also targets PDX1 in the cytoplasm). PDX1 then shuttles to the cytosol, where it is marked for ubiquitination and subsequent degradation by proteasome machinery, and beta cell function is impaired. PDX1 degradation itself, as well as loss of insulin, are further stimuli for apoptosis (adapted with permission from [5]) pro-diabetic-induced MST1 activation and beta cell apoptosis, whereas suppression of PI3K-AKT signalling enhances MST1 activity and beta cell apoptosis. This suggests the potential existence of bi-directional crosstalk between MST1 and AKT such that they functionally antagonise each other. This antagonism between MST1 and AKT implies that the outcome of simultaneous stimulation of both pathways is determined by the extent and duration of the respective apoptotic stimulus. In this context, MST1 may shift the balance towards the predominance of stress-triggered JNK activation, resulting in the BIM-dependent mitochondrial apoptotic pathway, whereas AKT-mediated survival signals are suppressed.

\section{MST1-PDX1: a deleterious interaction compromising beta cell function and survival}

In addition to initiating and potentiating beta cell apoptosis, MST1 specifically targets beta cell function by phosphorylating PDX1, thereby promoting its degradation. PDX1 is not only a key factor in pancreas development and function, its activity is a main control system of probably all vital functions of the beta cell, including susceptibility to apoptosis in general. Indeed, homozygous mutations in PDX1 have been reported to be responsible for the pancreatic agenesis associated with neonatal diabetes $[29,30]$. Importantly, heterozygosity for the null mutation, and hence reduced PDX1 expression levels, affect insulin expression and secretion and predispose islets to apoptosis $[29,31,32]$. Mutations in the $P D X 1$ gene can predispose individuals to the development of maturityonset diabetes of the young (MODY4) [33]. Heterozygous knockout mice $\left(P d x I^{+/}\right)$are glucose intolerant, with increased islet apoptosis, decreased islet mass and abnormal islet architecture, indicating that the appropriate gene dosage of $P d x 1$ is crucial for normal glucose homeostasis [32, 34]. PDX1 controls the expression of several genes that are vital for beta cell function (see Fig. 1). It directly binds to their promoters and enhances the expression of various beta cell genes, such as those encoding insulin, GLUT2 and glucokinase [29, 35]. PDX1 plays an essential role in the regulation of beta cell survival and, perhaps, susceptibility to apoptosis $[32,36$, 37]. PDX 1 deficiency contributes to diabetes progression through impaired proliferation and enhanced apoptosis via transcriptional mechanisms in different models of type 2 diabetes, such as Psammomys obesus and the $d b / d b$ mouse [38]. In turn, overexpression of PDX1 restores beta cell mass and function, thereby preventing the onset of diabetes in $\operatorname{Irs} 2$ knockout mice, confirming the critical role of PDX1 in beta cell survival [37]. Beta cell PDX1 expression is reduced in cellular models of glucose toxicity and accompanies the 
development of diabetes in complex genetic and environmental animal models of the disease, where low PDX1 levels correlate with beta cell failure [39].

PDX1 regulation is controlled by complicated signalling networks. At least three different post-translational modifications, including phosphorylation, sumoylation and glycosylation, have been suggested to explain nuclear-cytoplasmic shuttling and/or other functions of PDX1 [40-46]. Phosphorylation is a common post-translational modification implicated in the regulation of numerous transcription factors, but the precise role of PDX1 phosphorylation in the regulation of its subcellular localisation and activity remains unknown $[40,41,47]$. Short-term elevations in glucose levels promote the phosphorylation and subsequent nuclear translocation of PDX1 through one or a combination of several pathways, including the stress-activated protein kinase (SAPK), extracellular signal-regulated kinase (ERK) $1 / 2$ and PI3K-AKT pathways $[40,41,48]$. In contrast, the phosphorylation of specific residues within PDX1 negatively regulates its function. For example, phosphorylation of Ser-61 and Ser-66 of PDX1 via glycogen synthase kinase 3 (GSK3) appears to mark PDX1 for proteasomal degradation under conditions of oxidative stress [42].

All these studies indicate that intracellular PDX1 levels are critically involved in the regulation of cellular homeostasis, and that regulation is achieved by controlling the beta cell transcriptional program required for beta cell survival and function, especially when the cell is under stress. Therefore, the mechanism of PDX1 regulation and the functional consequences of altered PDX1 levels in the beta cell is critical and needs to be investigated in depth in the context of the progression of beta cell failure. While we found that PDXI mRNA levels were not affected by MST1 overexpression, PDX1 protein levels were heavily downregulated in beta cells, suggesting post-transcriptional regulation of PDX1 [5]. Conversely, MST1 silencing abolishes diabetic milieu-induced PDX1 downregulation, indicating the MST1-dependent PDX1 regulation in beta cells. Indeed, our further experiments uncovered that MST1 de-stabilises PDX1.

When PDX1 was introduced as a novel beta cell-specific substrate for MST1 we observed that the ubiquitination and subsequent degradation of PDX1 resulting from inhibitory Thr-11 phosphorylation is crucial for beta cell dysfunction in the setting of MST1 hyper-activation on top of the proapoptotic function of MST1. Thr-11-phosphorylation of PDX1 by MST1 marks PDX1 for degradation by the proteasome machinery, which would prohibit its function as a transcription factor in the nucleus. In this regard, overexpression of MST1 reduces the expression of PDX1 target genes and beta cell functional impairment, assessed by insulin secretion studies. Mutation of the Thr-11 site to alanine allowed PDX1 to be more stable, with less ubiquitination, and PDX1 transcriptional activity was maintained and beta cell function improved.
These observations establish the Thr-11 site of PDX1 as another potent target for beta cell rescue. Consistent with our observation, beta cell failure following DNA damage might be explained by the Thr-11 phosphorylation of PDX1 by DNAdependent protein kinases [43], the same site that is phosphorylated by MST1 (Fig. 1). Therefore, MST1 is a PDX1 kinase, and phosphorylation of PDX1 plays a very important role in regulating its function. MST1 deletion in beta cells is able to restore PDX1 levels even under diabetogenic conditions. Thus, while MST1 reduces beta cell survival, this effect is paralleled with a specific loss of function.

\section{Translation to diabetes therapy: outlook}

Taken together, our work provides evidence that MST1 acts as an essential apoptotic molecule that modulates diverse signalling pathways leading to beta cell apoptosis, as well as impaired beta cell insulin production and function. Inhibition of the MST1 pathway could thus represent an important strategy to rescue beta cell function and survival. To date, this strategy is working out well: potent inhibitors of MST1 have been identified (M. Tremblay [Calibr, La Jolla, CA, USA], A. Ardestani, K. Maedler; unpublished results) that have shown efficacy in preventing apoptosis in human islets and restoring glycaemia and beta cell mass in an animal model of diabetes, confirming previous results with Mstl knockout mice. The inhibitors are currently being tested in several animal models of diabetes, and we hope to move forward, always mindful of our ultimate goal: to make a better beta cell.

Funding Work by the authors is funded by the JDRF, the European Foundation for the Study of Diabetes (EFSD), Deutsche Forschungsgemeinschaft/German Research Foundation (DFG), the German Center for Diabetes Research (DZD) and the European Research Council (ERC).

Duality of interest The authors declare that there is no duality of interest associated with this manuscript.

Contribution statement Both authors were responsible for drafting the article and revising it critically for important intellectual content. Both authors approved the version to be published.

\section{References}

1. Ritzel RA, Butler AE, Rizza RA, Veldhuis JD, Butler PC (2006) Relationship between beta cell mass and fasting blood glucose concentration in humans. Diabetes Care 29:717-718

2. Herold KC, Usmani-Brown S, Ghazi T et al (2015) Beta cell death and dysfunction during type 1 diabetes development in at-risk individuals. J Clin Invest 125:1163-1173

3. Butler AE, Janson J, Bonner-Weir S, Ritzel R, Rizza RA, Butler PC (2003) Beta cell deficit and increased beta cell apoptosis in humans with type 2 diabetes. Diabetes 52:102-110 
4. Mathis D, Vence L, Benoist C (2001) Beta cell death during progression to diabetes. Nature 414:792-798

5. Ardestani A, Paroni F, Azizi Z et al (2014) MST1 is a key regulator of beta cell apoptosis and dysfunction in diabetes. Nat Med 20:385-397

6. Huang J, Wu S, Barrera J, Matthews K, Pan D (2005) The Hippo signaling pathway coordinately regulates cell proliferation and apoptosis by inactivating Yorkie, the Drosophila homolog of YAP. Cell 122:421-434

7. Zhao B, Li L, Lei Q, Guan KL (2010) The Hippo-YAP pathway in organ size control and tumorigenesis: an updated version. Genes Dev 24:862-874

8. Harvey KF, Zhang X, Thomas DM (2013) The Hippo pathway and human cancer. Nat Rev Cancer 13:246-257

9. Tremblay AM, Missiaglia E, Galli GG et al (2014) The Hippo transducer YAP1 transforms activated satellite cells and is a potent effector of embryonal rhabdomyosarcoma formation. Cancer Cell 26:273-287

10. Lin Z, von Gise A, Zhou P et al (2014) Cardiac-specific YAP activation improves cardiac function and survival in an experimental murine MI model. Circ Res 115:354-363

11. Barry ER, Camargo FD (2013) The Hippo superhighway: signaling crossroads converging on the Hippo/Yap pathway in stem cells and development. Curr Opin Cell Biol 25:247-253

12. Ma X, Chen $\mathrm{Y}, \mathrm{Xu} \mathrm{W}$ et al (2015) Impaired Hippo signaling promotes Rho1-JNK-dependent growth. Proc Natl Acad Sci U S A 112:1065-1070

13. Tumaneng K, Schlegelmilch K, Russell RC et al (2012) YAP mediates crosstalk between the Hippo and $\mathrm{PI}(3) \mathrm{K}-\mathrm{TOR}$ pathways by suppressing PTEN via miR-29. Nat Cell Biol 14:1322-1329

14. Avruch J, Zhou D, Fitamant J, Bardeesy N, Mou F, Barrufet LR (2012) Protein kinases of the Hippo pathway: regulation and substrates. Semin Cell Dev Biol 23:770-784

15. Radu M, Chernoff J (2009) The DeMSTification of mammalian Ste20 kinases. Curr Biol 19:R421-425

16. Graves JD, Draves KE, Gotoh Y, Krebs EG, Clark EA (2001) Both phosphorylation and caspase-mediated cleavage contribute to regulation of the Ste20-like protein kinase Mst1 during CD95/Fasinduced apoptosis. J Biol Chem 276:14909-14915

17. Lee KK, Ohyama T, Yajima N, Tsubuki S, Yonehara S (2001) MST, a physiological caspase substrate, highly sensitizes apoptosis both upstream and downstream of caspase activation. J Biol Chem 276:19276-19285

18. Ura S, Masuyama N, Graves JD, Gotoh Y (2001) Caspase cleavage of MST1 promotes nuclear translocation and chromatin condensation. Proc Natl Acad Sci U S A 98:10148-10153

19. Kurokawa M, Kornbluth S (2009) Caspases and kinases in a death grip. Cell 138:838-854

20. Cinar B, Fang PK, Lutchman M et al (2007) The pro-apoptotic kinase Mst1 and its caspase cleavage products are direct inhibitors of Akt1. EMBO J 26:4523-4534

21. Yuan T, Awal S, Kerr-Conte J, Ardestani A (2015) The hippo kinase LATS2 impairs pancreatic beta cell survival and function. Diabetologia 58: S46-S47 (Abstract)

22. Bonny C, Oberson A, Negri S, Sauser C, Schorderet DF (2001) Cell-permeable peptide inhibitors of JNK: novel blockers of beta cell death. Diabetes 50:77-82

23. Kaneto H, Nakatani Y, Miyatsuka T et al (2004) Possible novel therapy for diabetes with cell-permeable JNK-inhibitory peptide. Nat Med 10:1128-1132

24. Aikin R, Maysinger D, Rosenberg L (2004) Cross-talk between phosphatidylinositol 3-kinase/AKT and c-jun $\mathrm{NH}_{2}$-terminal kinase mediates survival of isolated human islets. Endocrinology $145: 4522-4531$
25. Tuttle RL, Gill NS, Pugh W et al (2001) Regulation of pancreatic beta cell growth and survival by the serine/threonine protein kinase Akt1/PKB $\alpha$. Nat Med 7:1133-1137

26. Hennessy BT, Smith DL, Ram PT, Lu Y, Mills GB (2005) Exploiting the PI3K/AKT pathway for cancer drug discovery. Nat Rev Drug Discov 4:988-1004

27. Jang SW, Yang SJ, Srinivasan S, Ye K (2007) Akt phosphorylates MstI and prevents its proteolytic activation, blocking FOXO3 phosphorylation and nuclear translocation. J Biol Chem 282:30836-30844

28. Yuan Z, Kim D, Shu S et al (2010) Phosphoinositide 3-kinase/Akt inhibits MST1-mediated pro-apoptotic signaling through phosphorylation of threonine 120. J Biol Chem 285:3815-3824

29. McKinnon CM, Docherty K (2001) Pancreatic duodenal homeobox-1, PDX-1, a major regulator of beta cell identity and function. Diabetologia 44:1203-1214

30. Stoffers DA, Zinkin NT, Stanojevic V, Clarke WL, Habener JF (1997) Pancreatic agenesis attributable to a single nucleotide deletion in the human IPF1 gene coding sequence. Nat Genet 15:106-110

31. Brissova M, Shiota M, Nicholson WE et al (2002) Reduction in pancreatic transcription factor PDX-1 impairs glucose-stimulated insulin secretion. J Biol Chem 277:11225-11232

32. Johnson JD, Ahmed NT, Luciani DS et al (2003) Increased islet apoptosis in $\mathrm{Pdx}^{+/-}$mice. $\mathrm{J}$ Clin Invest 111:1147-1160

33. Stoffers DA, Ferrer J, Clarke WL, Habener JF (1997) Early-onset type-II diabetes mellitus (MODY4) linked to IPF1. Nat Genet 17:138-139

34. Claiborn KC, Sachdeva MM, Cannon CE, Groff DN, Singer JD, Stoffers DA (2010) Pcifl modulates Pdx1 protein stability and pancreatic beta cell function and survival in mice. J Clin Invest 120:3713-3721

35. Babu DA, Deering TG, Mirmira RG (2007) A feat of metabolic proportions: Pdx1 orchestrates islet development and function in the maintenance of glucose homeostasis. Mol Genet Metab 92:43-55

36. Fujimoto K, Ford EL, Tran H et al (2010) Loss of Nix in Pdx1deficient mice prevents apoptotic and necrotic beta cell death and diabetes. J Clin Invest 120:4031-4039

37. Kushner JA, Ye J, Schubert M et al (2002) Pdx1 restores beta cell function in Irs2 knockout mice. J Clin Invest 109:1193-1201

38. Leibowitz G, Ferber S, Apelqvist A et al (2001) IPF1/PDX1 deficiency and beta cell dysfunction in Psammomys obesus, an animal with type 2 diabetes. Diabetes 50:1799-1806

39. Fujimoto K, Hanson PT, Tran H et al (2009) Autophagy regulates pancreatic beta cell death in response to Pdx1 deficiency and nutrient deprivation. J Biol Chem 284:27664-27673

40. Elrick LJ, Docherty K (2001) Phosphorylation-dependent nucleocytoplasmic shuttling of pancreatic duodenal homeobox-1. Diabetes 50:2244-2252

41. Macfarlane WM, McKinnon CM, Felton-Edkins ZA, Cragg H, James RF, Docherty K (1999) Glucose stimulates translocation of the homeodomain transcription factor PDX1 from the cytoplasm to the nucleus in pancreatic beta-cells. J Biol Chem 274:1011-1016

42. Boucher MJ, Selander L, Carlsson L, Edlund H (2006) Phosphorylation marks IPF1/PDX1 protein for degradation by glycogen synthase kinase 3-dependent mechanisms. J Biol Chem 281:6395-6403

43. Lebrun P, Montminy MR, van Obberghen E (2005) Regulation of the pancreatic duodenal homeobox-1 protein by DNA-dependent protein kinase. J Biol Chem 280:38203-38210

44. Kishi A, Nakamura T, Nishio Y, Maegawa H, Kashiwagi A (2003) Sumoylation of $\mathrm{Pdx} 1$ is associated with its nuclear localization and insulin gene activation. Am J Physiol Endocrinol Metab 284:E830-E840 
45. Gao Y, Miyazaki J, Hart GW (2003) The transcription factor PDX-1 is post-translationally modified by $O$-linked $N$-acetylglucosamine and this modification is correlated with its DNA binding activity and insulin secretion in min6 beta-cells. Arch Biochem Biophys 415:155-163

46. An R, da Silva XG, Semplici F et al (2010) Pancreatic and duodenal homeobox 1 (PDX1) phosphorylation at serine-269 is HIPK2dependent and affects PDX1 subnuclear localization. Biochem Biophys Res Commun 399:155-161
47. Kawamori D, Kaneto H, Nakatani Y et al (2006) The forkhead transcription factor Foxol bridges the JNK pathway and the transcription factor PDX-1 through its intracellular translocation. J Biol Chem 281:1091-1098

48. Rafiq I, Kennedy HJ, Rutter GA (1998) Glucose-dependent translocation of insulin promoter factor-1 (IPF-1) between the nuclear periphery and the nucleoplasm of single MIN6 beta-cells. J Biol Chem 273:23241-23247 